\title{
JUCYS-MURPHY ELEMENTS AND THE UNITARY WEINGARTEN FUNCTION
}

\author{
JONATHAN I. NOVAK \\ Combinatorics $\&$ Optimization, University of Waterloo \\ 200 University Avenue West, Waterloo, Ontario, Canada N2L $3 G 1$ \\ E-mail:j2novak@math.uwaterloo.ca
}

\begin{abstract}
We describe an approach to the unitary Weingarten function based on the JM elements of symmetric group algebras. When combined with previously known properties of the Weingarten function, this gives a surprising connection with the Moebius function of the lattice of noncrossing partitions.
\end{abstract}

\section{Introduction}

1.1. Moments of the unitary group. Let $\mathbb{U}_{d}=\left\{U \in G L_{d}(\mathbb{C}): U^{*}=U^{-1}\right\}$ be the compact group of $d \times d$ complex unitary matrices equipped with (normalized) Haar measure $d U$. In the context of random matrix theory, one often wishes to compute integrals (expectations) of the form

$$
I_{d}\left(i, j, i^{\prime}, j^{\prime}\right)=\int_{\mathbb{U}_{d}} u_{i(1) j(1)} \ldots u_{i(n) j(n)} \bar{u}_{i^{\prime}(1) j^{\prime}(1)} \ldots \bar{u}_{i^{\prime}(n) j^{\prime}(n)} d U,
$$

where $n$ is a positive integer and $i, j, i^{\prime}, j^{\prime}:[n] \rightarrow[d]$ are functions.

Integrals of this form, which describe the joint moments of the entries of a Haardistributed random unitary matrix from $\mathbb{U}_{d}$, were studied extensively by Collins [C] and Collins-Śniady [CS], where they were called the moments of the unitary group $\mathbb{U}_{d}$. The computation of these moments is ostensibly a probabilistic problem; however, the fact that $\mathbb{U}_{d}$ is a compact algebraic group allows for an essentially algebraic solution.

1.2. The Weingarten function. We assume henceforth that $d \geq n$. This assumption simplifies the presentation of some results concerning unitary group integrals. It

2000 Mathematics Subject Classification: Primary 05E05, 05E10; Secondary 15B52.

Key words and phrases: Jucys-Murphy elements, Weingarten function, symmetric functions, Haar unitary random matrices.

The paper is in final form and no version of it will be published elsewhere. 
was shown in [CS] that, in most cases, this assumption can be lifted without too much difficulty.

The main result of Collins in $\left[\mathrm{C}\right.$ is that there exists a function $\mathrm{Wg}_{d}: S(n) \rightarrow \mathbb{C}$, where $S(n)$ is the symmetric group on $[n]:=\{1, \ldots, n\}$, with the following remarkable property:

$$
I_{d}\left(i, j, i^{\prime}, j^{\prime}\right)=\sum_{\sigma, \tau \in S(n)} \prod_{k=1}^{n} \delta_{i(k), i^{\prime}(\sigma(k))} \delta_{j(k), j^{\prime}(\sigma(k))} \mathrm{Wg}_{d}\left(\tau \sigma^{-1}\right),
$$

where $\delta$ is the Kronecker delta. The special function $\mathrm{Wg}_{d}$ is called the Weingarten function, and formula (2) above is the Weingarten convolution formula. The existence of the Weingarten function was deduced by Collins from the classical Schur-Weyl duality between representations of $\mathbb{U}_{d}$ and $S(n)$ in the tensors

$$
\underbrace{\mathbb{C}^{d} \otimes \mathbb{C}^{d} \otimes \cdots \otimes \mathbb{C}^{d}}_{n}
$$

The problem of computing the integrals (1) is thus reduced, via the Weingarten convolution formula (2), to the problem of finding a tractable expression for the Weingarten function.

1.3. Main result. Since $\mathrm{Wg}$ is a function on permutations, we view it as an element of the symmetric group algebra $\mathbb{C}[S(n)]$, i.e. as a formal sum

$$
\mathrm{Wg}_{d}=\sum_{\sigma \in S(n)} \mathrm{Wg}_{d}(\sigma) \sigma .
$$

In this note we show that a remarkably simple formula for $\mathrm{Wg}_{d}$ can be given in terms of the Jucys-Murphy elements of $\mathbb{C}[S(n)]$ :

$$
\begin{aligned}
J_{1} & =0, \\
J_{2} & =(1,2), \\
J_{2} & =(1,3)+(2,3), \\
& \vdots \\
J_{n} & =(1, n)+(2, n)+\cdots+(n-1, n) .
\end{aligned}
$$

Our main theorem is the following:

TheOREM 1.1. Let $d \geq n$ be positive integers. Then $d+J_{1}, d+J_{2}, \ldots, d+J_{n} \in \mathbb{C}[S(n)]$ are invertible, and

$$
\mathrm{Wg}_{d}=\left(d+J_{1}\right)^{-1}\left(d+J_{2}\right)^{-1} \ldots\left(d+J_{n}\right)^{-1} \text {. }
$$

\section{Proofs}

2.1. Background on JM elements. The JM elements $J_{1}, \ldots, J_{n} \in \mathbb{C}[S(n)]$ were introduced independently by Jucys [J] and Murphy [M]. These seemingly simple transposition sums have many remarkable properties, and are a key object in the modern approach 
to the representation theory of the symmetric groups initiated by Okounkov and Vershik [OV]. In particular, the JM elements generate a maximal commutative subalgebra of $\mathbb{C}[S(n)]$.

Let $Z(n)$ denote the center of $\mathbb{C}[S(n)]$. Let $\mathbb{Y}(n)$ be the set of Young diagrams with $n$ cells, and recall the canonical surjection $T: S(n) \rightarrow \mathbb{Y}(n)$ which maps each permutation to its cycle type. Given $\mu \in \mathbb{Y}(n)$, let $\mathcal{C}_{\mu}$ denote the fibre of $T$ over $\mu$, i.e. the conjugacy class of permutations of cycle type $\mu$, which we identify with the sum

$$
\mathcal{C}_{\mu}=\sum_{T(\sigma)=\mu} \sigma \in Z(n) .
$$

We also denote by $\ell(\mu)$ the number of rows in a Young diagram $\mu$. In particular, $\ell(T(\sigma))$ is the number of factors in the decomposition of $\sigma$ into a product of disjoint cycles.

We will benefit greatly from the following result of Jucys:

TheOREM $2.1([\bar{J}])$. For $1 \leq r \leq n$, let

$$
e_{r}\left(J_{1}, J_{2}, \ldots, J_{n}\right)=\sum_{1 \leq i_{i}<i_{2}<\cdots<i_{r} \leq n} J_{i_{1}} J_{i_{2}} \ldots J_{i_{r}}
$$

be the elementary symmetric polynomials in JM elements. Then

$$
e_{r}\left(J_{1}, J_{2}, \ldots, J_{m}\right)=\sum_{\substack{\mu \in \mathbb{Y}(n) \\ \ell(\mu)=n-r}} \mathcal{C}_{\mu} .
$$

In other words, $e_{r}\left(J_{1}, J_{2}, \ldots, J_{n}\right)$ is the indicator function of permutations with exactly $n-r$ cycles.

For example,

$$
e_{1}\left(J_{1}, J_{2}, \ldots, J_{n}\right)=J_{1}+J_{2}+\cdots+J_{n}
$$

is the formal sum of all permutations with exactly $n-1$ cycles (i.e. the conjugacy class of transpositions).

2.2. Proof of Theorem 1.1. Our starting point is the following fundamental theorem of Collins [C]: for $d \geq n$, the inverse of $\mathrm{Wg}_{d}$ is

$$
\mathrm{Wg}_{d}^{-1}=\sum_{\mu \in \mathbb{Y}(n)} d^{\ell(\mu)} \mathcal{C}_{\mu}
$$

Now we simply compute using Jucys' Theorem 2.1

$$
\begin{aligned}
\left(d+J_{1}\right)\left(d+J_{2}\right) \ldots\left(d+J_{n}\right) & =\sum_{k=0}^{n} d^{n-k} e_{k}\left(J_{1}, J_{2}, \ldots, J_{n}\right) \\
& =\sum_{k=0}^{n} \sum_{\substack{\mu \in \mathbb{Y}(n) \\
\ell(\mu)=n-k}} d^{n-k} \mathcal{C}_{\mu} \\
& =\sum_{\mu \in \mathbb{Y}(n)} d^{\ell(\mu)} \mathcal{C}_{\mu} .
\end{aligned}
$$


It follows immediately from the assumption $d \geq n$ that $d+J_{k}$ is invertible for each $1 \leq k \leq n$. Thus

$$
\mathrm{Wg}_{d}=\left(d+J_{1}\right)^{-1}\left(d+J_{2}\right)^{-1} \ldots\left(d+J_{n}\right)^{-1},
$$

as desired.

3. Application: the Laurent expansion. Consider a fixed permutation $\sigma \in S(n)$ with cycle type $\tau=\left(\tau_{1}, \tau_{2}, \ldots, \tau_{\ell}\right)$. $\mathrm{Wg}_{d}(\sigma)$ is then a rational function of $d$ with denominator of degree $n[\mathrm{C}$. Thus there exists a Laurent expansion

$$
\mathrm{Wg}_{d}(\sigma)=\sum_{r \geq 0} \frac{a_{r}(\sigma)}{d^{n+r}} .
$$

The structure of the Laurent coefficients remains somewhat mysterious. Collins [C] has shown that

$$
\min \left\{r: a_{r}(\sigma) \neq 0\right\}=n-\ell
$$

and moreover

$$
a_{n-\ell}(\sigma)=\operatorname{Moeb}(\sigma) .
$$

Recall that Moeb is the Moebius function defined by

$$
\operatorname{Moeb}(\sigma)=\prod_{k=1}^{\ell}(-1)^{\tau_{k}-1} \operatorname{Cat}_{\tau_{k-1}}
$$

where

$$
\text { Cat }_{m}=\frac{1}{m+1}\left(\begin{array}{c}
2 m \\
m
\end{array}\right)
$$

is a Catalan number. Moeb coincides with the Moebius function of the lattice of noncrossing partitions, see [NS].

The existence of the Laurent expansion 15 means that there exists a sequence of functions $a_{0}, a_{1}, \ldots, a_{r}, \cdots \in \mathbb{C}[S(n)]$ such that, if we define

$$
H_{N}=\sum_{r=0}^{N} \frac{a_{r}}{d^{n+r}}
$$

then

$$
\mathrm{Wg}_{d}(\sigma)=\lim _{N \rightarrow \infty} H_{N}(\sigma)
$$

for all $\sigma \in S(n)$ (where we are assuming $d \geq n$ ).

THEOREM 3.1. For each $r \geq 0$,

$$
a_{r}=(-1)^{r} h_{r}\left(J_{1}, J_{2}, \ldots, J_{n}\right),
$$

where

$$
h_{r}\left(J_{1}, J_{2}, \ldots, J_{n}\right)=\sum_{1 \leq i_{1} \leq i_{2} \leq \cdots \leq i_{r} \leq n} J_{i_{1}} J_{i_{2}} \ldots J_{i_{n}}
$$

is the rth complete homogeneous symmetric polynomial in the JM elements (i.e. simply the sum of all distinct monomials of degree $r$ ). 
Proof. If $x_{1}, \ldots, x_{n}, t$ are formal indeterminates, one has the generating function

$$
\sum_{r \geq 0} h_{r}\left(x_{1}, \ldots, x_{n}\right) t^{r}=\left(1-x_{1} t\right)^{-1}\left(1-x_{2} t\right)^{-1} \ldots\left(1-x_{n} t\right)^{-1} .
$$

Simply specialize $x_{1}=J_{1}, x_{2}=J_{2}, \ldots, x_{n}=J_{n}$ and $t=-\frac{1}{d}$ and apply Theorem 1.1 to obtain the stated result.

One immediate consequence of this expression of $a_{r}$ in terms of the JM elements is the following:

$$
a_{0}(\sigma)= \begin{cases}1, & \text { if } \sigma=e, \\ 0, & \text { otherwise. }\end{cases}
$$

This is simply because $h_{0}\left(J_{1}, J_{2}, \ldots, J_{n}\right)$ is the unit element of the group algebra $\mathbb{C}[S(n)]$, i.e. the indicator function of the identity element.

Let us point out how remarkably this $h$-function result interacts with the work of Collins. Recall that the number $|\sigma|:=n-\ell(T(\sigma))$ is the minimal length of a factorization of $\sigma$ into transpositions. Our remarks above amount to the following striking result:

THEOREM 3.2. For any permutation $\sigma \in S(n)$,

$$
\operatorname{Moeb}(\sigma)=(-1)^{|\sigma|} h_{|\sigma|}\left(J_{1}, \ldots, J_{n}\right)(\sigma) .
$$

It should be possible to prove this remarkable fact using only intrinsic properties of JM elements, and this will be the subject of future work.

\section{References}

[C] B. Collins, Moments and cumulants of polynomial random variables on unitary groups, the Itzykson-Zuber integral, and free probability, International Mathematics Research Notices 17 (2003), 953-982.

[CS] B. Collins and P. Śniady, Integration with respect to the Haar measure on unitary, orthogonal, and symplectic group, Communications in Mathematical Physics 264 (2006), 773-795.

[J] A. Jucys, Symmetric polynomials and the center of the symmetric group ring, Reports on Mathematical Physics 5 (1974), 107-112.

[M] G. Murphy, A new construction of Young's seminormal representation of the symmetric group, Journal of Algebra 69 (1981), 287-291.

[NS] A. Nica and R. Speicher, Lectures on the Combinatorics of Free Probability, London Mathematical Society Lecture Note Series 335, Cambridge University Press, 2006

[OV] A. Okounkov and A. Vershik, A new approach to the representation theory of symmetric groups, Selecta Mathematica 4 (1996), 581-605. 
